\title{
Patterns, Sets of Outcomes, and Combinatorial Justification: Two Students' Reinvention of Counting Formulas
}

\author{
Elise Lockwood • Craig A. Swinyard • John S. Caughman
}

Published online: 14 April 2015

(C) Springer International Publishing Switzerland 2015

\begin{abstract}
Counting problems provide an accessible context for rich mathematical thinking, yet they can be surprisingly difficult for students. To foster conceptual understanding that is grounded in student thinking, we engaged a pair of undergraduate students in a ten-session teaching experiment. The students successfully reinvented four basic counting formulas, but their work revealed a number of unexpected issues concerning justification in counting. In this paper, we describe the students' successful reinvention of the four counting formulas, we critically examine their combinatorial reasoning in terms of Lockwood's (2013) model of students' combinatorial thinking, and we offer several directions for further research.
\end{abstract}

Keywords Combinatorics $\cdot$ Reinvention $\cdot$ Counting problems $\cdot$ Teaching experiment

\section{Introduction and Motivation}

Enumerative combinatorics (the solving of counting problems) has applications in probability and computer science, and its accessible yet challenging problems provide a rich context for developing students' mathematical justification and problem solving skills. As a result, counting problems have gained traction in K-12 and undergraduate curricula in recent years, particularly in probability units and in undergraduate discrete mathematics courses. Both researchers (e.g., Hadar and Hadass 1981; Maher, Powell,

\footnotetext{
E. Lockwood $(\bowtie)$

Department of Mathematics, Oregon State University, 338 Kidder Hall, Corvallis, OR 97331, USA

e-mail: elise314@gmail.com

C. A. Swinyard

Department of Mathematics, University of Portland, 214 Buckley Center, Portland, OR 97203, USA

e-mail: swinyard@up.edu

J. S. Caughman

Department of Mathematics, Portland State University, M-319 Neuberger Hall, Portland, OR 97201, USA

e-mail: caughman@pdx.edu
} 
and Uptegrove 2011) and textbook authors (e.g., Martin 2001; Tucker 2002) have noted that counting problems facilitate deep and critical mathematical reasoning. For example, Tucker (2002) emphasizes that counting requires "logical reasoning, clever insights, and mathematical modeling” (p. 169). Martin (2001) similarly points out, "One of the things that make elementary counting difficult is that we will encounter very few algorithms. You will have to think" (p. 1).

Often one of the first topics a student encounters in an undergraduate discrete mathematics course, counting offers a rich context for developing the type of mathematical justification skills necessary for advanced courses. However, in spite of the practical applications of counting problems and their potential to foster rich mathematical thinking, student difficulties with counting persist (e.g., Batanero, Navarro-Pelayo, and Godino 1997; Eizenberg and Zaslavsky 2004). More research is needed that explicates how students can effectively comprehend basic counting principles.

The aim of our study was to gain insight into how students might come to reason coherently about four basic counting formulas: $n !, n^{r}, \frac{n !}{(n-r) !}$, and $\frac{n !}{(n-r) ! r !}$. Textbooks typically present these formulas early on, following each with numerous examples, and students are generally expected to apply the formulas in various contexts throughout the remainder of the course. Research (e.g., Batanero, et al. 1997; Lockwood 2011a) indicates, however, that students frequently misapply these formulas, which suggests they may not understand when and why these expressions are to be used. Recent studies (Oehrtman, Swinyard, and Martin 2014; Swinyard 2011) suggest that students can develop coherent reasoning about mathematical concepts via tasks designed to foster their reinvention of precise concept definitions (Tall and Vinner 1981). These studies have also served as evidence that reinvention can provide researchers a lens through which to gain insight into how students come to understand particular mathematical concepts. With this in mind, we engaged a pair of undergraduates in a ten-session teaching experiment, during which they solved basic counting problems and then subsequently generalized their mathematical activity by reinventing the four basic counting formulas. In this paper, we report on the students' reinvention of these four formulas, addressing the following questions:

1) How might students reinvent these four basic counting formulas?

2) As students reinvent and use these formulas, what insights might we gain about their combinatorial thinking?

\section{Literature Review}

Student Difficulties with Counting Martin (2001) begins his advanced combinatorics textbook by stating, "Counting is hard" (p. 1). While this is a clever play on the difference between children's counting and combinatorial enumeration, Martin's point highlights perennial difficulties that students face. Those who teach counting also acknowledge the fact that students can struggle with learning counting. In fact, Annin and Lai (2010) claim that counting is the most difficult topic for them to teach (p. 403). Researchers have clearly established that students have trouble with even basic counting tasks, and 
Batanero et al. (1997) highlight the need for researchers to explore and better understand such difficulties:

Combinatorics is a field that most pupils find very difficult. Two fundamental steps for making the learning of this subject easier are understanding the nature of pupils' mistakes when solving combinatorial problems and identifying the variables that might influence this difficulty (p. 182).

One way in which these struggles can be seen is in overall low success rates on counting problems. For example, Eizenberg and Zaslavsky reported that among undergraduate students who had taken at least one course in combinatorics, only $40 \%$ (43 of 108 ) of initial solutions to counting problems were correct $(2004$, p. 31). Lockwood (2011a) similarly found that only 42 out of 103 (41\%) problems given to 22 postsecondary mathematics students were initially answered correctly, even during an interview setting in which the students had few time constraints. Godino, Batanero, and Roa (2005) also note that 118 undergraduate mathematics majors "generally found it difficult to solve the problems (each student only solved an average number of 6 [of 13] problems correctly)" (p. 4).

Some researchers have attempted to identify factors that might lead to difficulties for students. This has included drawing attention to pitfalls (Hadar and Hadass 1981) and highlighting common errors that students make, such as over-counting and confusing situations in which order matters or does not matter (e.g., Annin and Lai 2010; Batanero, et al. 1997). Additionally, Eizenberg and Zaslavsky (2004) and Lockwood (2014a) have pointed out the fact that counting problems, with their large numerical answers, can be difficult to verify. In efforts to ameliorate students' difficulties, some researchers have identified fruitful strategies that students may employ. For example, researchers have examined young children's (English, 1991, 1993) and undergraduates' (Lockwood and Gibson, 2014) listing strategies, Maher shows young students' sophisticated reasoning on combinatorial tasks (Maher, et al. 2011), and Lockwood (2011b) suggests that students can make unexpected and useful connections among counting situations. Others (e.g., Halani 2012; Lockwood 2013, 2014b) have extended such work to focus not only on strategies, but also on ways of thinking that might be productive for students. However, in spite of such studies, there is still much work to be done to address systematically students' issues with counting so that they might be well-supported in coming to reason coherently about counting.

Research on Combinatorial Concepts In our study, students reinvented formulas for basic combinatorial notions, including permutations and combinations. Our work builds on prior research, including that of Piaget and Inhelder (1975), who posited a fundamental difference between the mental processes that combinations and permutations each respectively require. Dubois (1984), Fischbein and Gazit (1988), and Batanero, et al. (1997) have also investigated the effects of both implicit combinatorial models and particular combinatorial operations on students' counting. Our study extends this prior work by utilizing reinvention to target undergraduate students' conceptualizations of permutations and combinations.

This paper is also framed within Lockwood's (2013) model of combinatorial thinking, in which she describes and relates three components of students' counting 
(formulas/expressions, counting processes, and sets of outcomes) and argues that students should focus on sets of outcomes as they count. The model is elaborated in the theoretical perspective section, which we present after a brief mathematical discussion.

\section{Mathematical Discussion - The Multiplication Principle}

The multiplication principle is considered by many (e.g., Martin 2001; Mazur 2009; Tucker 2002) to be a foundational aspect of counting - indeed, it is the basis for understanding and justifying counting formulas. Nonetheless, among textbooks there does not seem to be much consensus for a precise statement of the multiplication principle. Two statements are given below, the second of which offers a more general statement of the principle.

- "The Fundamental Principle of Counting: If one task can be completed in $m$ ways and another task can be completed in $n$ ways, then the sequence of the two tasks can be completed in $m \times n$ ways." (Richmond and Richmond 2009, p. 132)

- "The Multiplication Principle: Suppose a procedure can be broken into $m$ successive (ordered) stages, with $r_{1}$ different outcomes in the first stage, $r_{2}$ different outcomes in the second stage,..., and $r_{m}$ different outcomes in the $m^{\text {th }}$ stage. If the number of outcomes at each stage is independent of the choices in previous stages, and if the composite outcomes are all distinct, then the total procedure has $r_{1} \times r_{2} \times \ldots \times r_{m}$ different composite outcomes" (Tucker 2002, p. 170, emphasis in original).

We mention the multiplication principle here because it is foundational to basic counting in two key ways. First, it provides some justification for solutions to counting problems, and second, it underpins several basic counting formulas that students encounter. To illustrate the former, we consider the following problem:

Quiz Questions problem - On a quiz, there are eight multiple-choice questions, each of which has four possible answers (A, B, C, and D). In how many ways could a student complete this quiz? (We may assume that the student must answer all of the questions.)

To answer this question, we could make the following argument: We have four options for how to answer the first question - A, B, C, or D. Then, for any of those possibilities, we again have four options for how to answer the second question, yielding 16 possibilities for answering two questions. By the same argument, we will have four choices for each of the third through eighth questions, and we continue to multiply by four for each question. This is illustrated by a partial tree diagram in Fig. 1. While we could formally connect the answer to the problem with a rigorous statement of the multiplication principle, even the informal argument we have provided gives some insight and justification into the situation. The multiplication principle allows us to explain the general process that justifies why repeated multiplication by four is reasonable without having to list all $4^{8}$ (or 65,536 ) outcomes. 


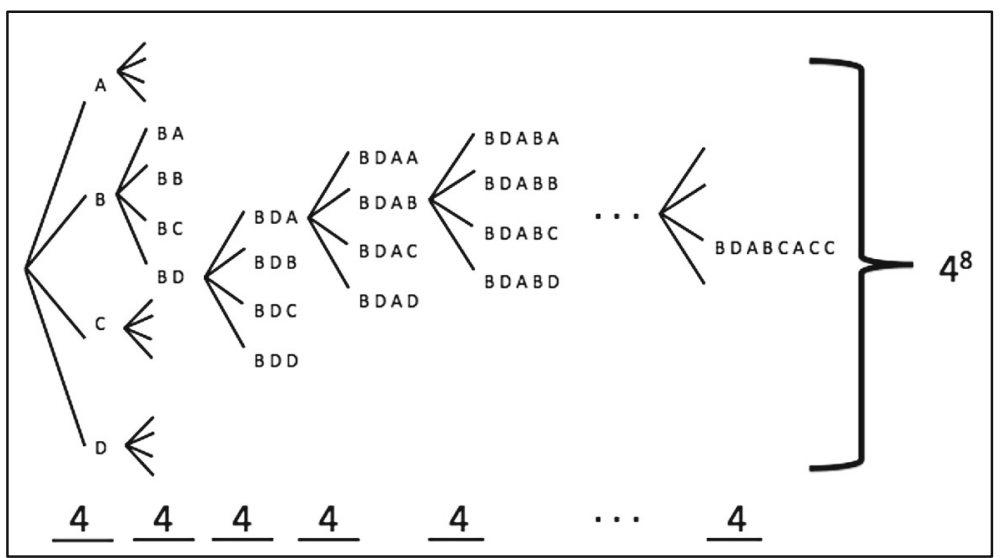

Fig. 1 A tree diagram representing the multiplication principle

Second, the multiplication principle is foundational to counting because it underpins several basic counting formulas students encounter. Formulas like $n !, n^{r}, \frac{n !}{(n-r) !}$, and $\frac{n !}{(n-r) ! r !}$ are each general ways to express particular products that are based on the multiplication principle. Specifically, $n$ ! is the number of ways to arrange $n$ objects when objects cannot be repeated. Indeed, if we picture placing objects in distinct, ordered positions, there are $n$ choices for which object goes in the first position, $n-1$ choices for the second position, and so on. Through the lens of the multiplication principle, this can be thought of as distinct, ordered stages, and thus it makes sense that the resulting product of $n$ ! gives all of the possible arrangements of $n$ objects. The formula $\frac{n !}{(n-r) !}$ is a variation of the $n$ ! formula - instead of arranging all $n$ objects, we may only want to arrange $r$ of $n$ objects. There is again simply a product (based on the multiplication principle) that we wish to write, and the formula provides an efficient way to express that product. The $n^{r}$ and $\frac{n !}{(n-r) !}$ formulas are similarly supported by the multiplication principle. Therefore, we see that the multiplication principle provides justification for our counting, thus conceptually supporting the vast majority of enumerative combinatorics, and it is foundational to much of the initial counting that students encounter. Throughout the paper we make reference to students' understanding of the multiplication principle, the counting formulas they reinvented, and the solutions for different tasks. By "understanding" we mean the knowledge that they communicate about a concept, and we think of conceptual understanding as being in line with Hiebert and Lefevre's (1986) conceptual knowledge.

\section{Theoretical Perspective}

A Model of Students' Combinatorial Thinking Our work is situated within the context of Lockwood's (2013) model of students' combinatorial thinking (Fig. 2), which we elaborate here, using the Quiz Questions problem as an example. The model consists of three components - formulas/expressions, counting processes, and sets of outcomes. The formulas/expressions are mathematical expressions that yield some numerical 


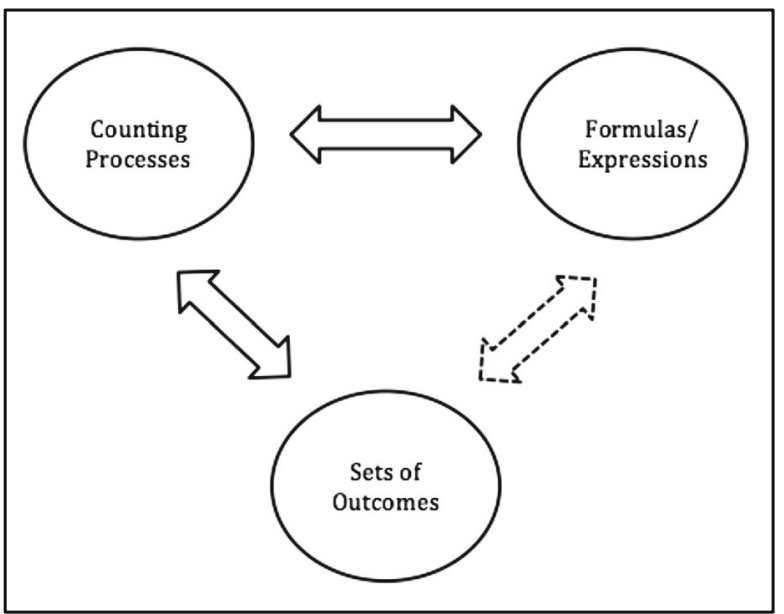

Fig. 2 Lockwood's (2013) model of students' combinatorial thinking

value, and are often considered "the answer" to the counting problem (p. 252). In the Quiz Questions problem, an expression that gives the final answer is $4^{8}$. The counting processes are the enumeration processes (or series of processes) in which a counter engages as they solve a counting problem (p. 253). In the Quiz Questions problem, the counting process is the iterative use of the multiplication principle to track the options at each stage. The set of outcomes refers to the set of elements that one can imagine being generated or enumerated by a counting process (p. 253). In our example, the set of outcomes consists of the collection of all the ways in which a quiz might be answered, perhaps encoded as sequences of length 8 of the letters A, B, C, or D. The cardinality of the set of outcomes $(65,536$ in this case) is regarded as the problem's solution.The model also elaborates relationships between the three components and emphasizes the importance of fostering the relationship between counting processes and sets of outcomes. In the initial conceptualization of the model, it was not fully explored what the direct relationship between formulas/expressions and sets of outcomes might entail, as evidenced by the dotted arrow. Despite a few conjectures, there was simply not yet enough empirical data to substantiate or articulate the relationship. As such, the model initially served primarily to highlight sets of outcomes as a central construct underlying the counting processes and formulas with which students interact. Additionally, the model can help us characterize students' typical counting practice - they are inclined not to think about sets of outcomes as they count, but rather they often rely on memorized key words or situations to determine whether order matters or to decide which formula to apply (e.g., Annin and Lai 2010; Lockwood 2014a, 2014b). In previous work (e.g., Lockwood 2011a) we have often seen students operate with numbers in a problem without considering whether their answers make sense in terms of the outcomes that are being counted. For example, in the Quiz Questions problem, students may arrive at an answer of $4+4+4+4+4+4+4+$ $4=4 \times 8$, without being able to justify this expression, or see why that answer would not provide all of the desirable outcomes of the counting problem. While some simple problems can be solved without considering outcomes (such as correctly employing multiplication to arrive at an answer of $4^{8}$ for the Quiz Questions problem), using 
outcomes can resolve many commonly troublesome aspects of counting (such as issues of order or reconciling an overcount). Previous work (Lockwood 2011a, 2013; 2014b) has shown that students tend not to utilize outcomes as often as they could. The model thus provides language through which to articulate several salient aspects of students' counting activity.

Developmental Research In designing the present teaching experiment, we conjectured, based on prior research (Lockwood 2011a), that students would have a better chance of successfully using counting formulas (and solving counting problems successfully) if the counting formulas contain meaning for them. Given that our central research goal was to gain insight into how students might reason meaningfully about the four counting formulas previously discussed, we chose not to supply them with these formulas, but rather to have them reinvent the formulas (i.e., construct the formulas themselves) by generalizing their work on an initial set of counting problems. We patterned our work after previous studies (Oehrtman, et al. 2014; Swinyard 2011) in which students were able to develop coherent reasoning about mathematical concepts via tasks designed to foster their reinvention of precise mathematical definitions. We thus drew inspiration from the perspective of developmental research (Gravemeijer 1998), which leverages students' informal knowledge and supports them in developing sophisticated, abstract knowledge while maintaining intellectual autonomy (p. 279). In line with Freudenthal's recommendation (1973) to avoid an antididactic inversion (where symbolic formalism precedes reasoning), we aimed to create an environment that fosters initial exploration of counting problems that emphasizes sense-making over conventional symbolization.

\section{Methods}

The aim of this paired teaching experiment (Steffe and Thompson 2000) was for two students to reinvent four basic counting formulas through engaging with a variety of counting problems.

Participants The participants were two above-average students (Thomas and Robin, pseudonyms) who had recently completed an integral calculus course at a university in the Northwestern United States. They were chosen based on the following criteria: 1) they had not taken an undergraduate, university-level course for which counting was included as a topic (such as a discrete mathematics, probability, or combinatorics course); 2) they demonstrated strong mathematical background and ability; and, 3) they displayed a propensity to engage actively with mathematics and articulate their reasoning, as observed by one of the authors during the integral calculus course. The teaching experiment occurred following Thomas and Robin's freshman and sophomore years, respectively.

Data Collection and Tasks The paired teaching experiment consisted of ten 90-minute sessions, which occurred three times a week for about four weeks. The sessions were conducted in a classroom, with the two students primarily working on counting 
problems on the whiteboard in the front of the room. Only the participating students and two researchers (the first and second authors) were present for each session. We point out that the tasks in the teaching experiment were specifically chosen to help the students follow a targeted progression toward reinvention of the four previously discussed formulas, but in their work on the tasks the students generally worked independently of the interviewers and interacted primarily with each other. The interviewers at times asked questions of clarification to gain insight into what the students were thinking, and, similar to previous reinvention studies (e.g., Oehrtman, et al. 2014), the interviewers intervened only when the students had stopped working productively on their own. During such interventions, which were infrequent, the interviewers prompted the students to think about aspects of their prior work that might help them proceed in a productive manner. It is also worth noting that the students decided when they were finished with a problem; the interviewers gave no indication that the students should stop working or that they had arrived at a correct (or incorrect) answer. The teaching experiment consisted of three phases, which we describe in detail.

Phase 1 (Sessions 1-4): Solving Initial Counting Problems Since neither student had prior counting experience, we first had them solve ten counting problems, with the goal of providing a common experience from which to generalize their mathematical activity. Three factors played into our task selection. First, we wanted Thomas and Robin primarily to engage in problem solving. Although these students had no formal collegiate experience with counting, we recognized that they may have seen permutation or combination formulas in high school. To encourage problem solving rather than merely trigger attempts to recall expressions, we included some initial problems that could not be solved with one direct application of the most basic formulas. Second, we wanted students to use and reason about sets of outcomes, and thus most of these initial problems involved numbers small enough to facilitate explicit listing. ${ }^{1}$ Finally, we aimed for a variety of problems to require a range of approaches. This included problems that might naturally be broken into cases, problems for which the multiplication principle might naturally be applied, and at least a few problems with varying sets of outcomes. We provide the Phase 1 tasks in Table 1.

Phase 2 (Sessions 5-7): Reinventing Counting Formulas Our aim in Phase 2 was to see if the students could reinvent each of the four basic counting formulas. To foster this reinvention, we chose tasks that we felt would motivate generalization, in that the solution sets are too large to enumerate easily via listing. In Table 2, we provide the tasks associated with the reinvention of each formula. We used a common context (a basketball game) for the last two tasks to provide an opportunity to generate and leverage natural connections between permutations and combinations. To count all combinations, we could first count permutations (as all possible arrangements of $r$ objects from $n$ objects) and divide by $r$ !, as each set of $r$ objects is actually getting counted $r$ ! times. ${ }^{2}$ Given this relationship, the problems we chose involving

\footnotetext{
${ }^{1}$ The obvious exception is the Quiz Questions problem, but prior experience with this problem suggested that it would be accessible to the students.

${ }^{2}$ Conversely, the number of permutations of $r$ objects from $n$ objects can be thought of as first selecting $r$ objects (there are $n$ choose $r$ ways to do this), and then permuting the $r$ objects (in $r$ ! ways).
} 
Table 1 Phase 1 tasks - Solving initial counting problems

Problem Name Problem Statement

\begin{tabular}{|c|c|}
\hline Dominos & $\begin{array}{l}\text { A domino is a small, thin rectangular tile that has dots on one of its broad faces. That face } \\
\text { is split into two halves, and there can be zero through six dots on each of those halves. } \\
\text { Suppose you want to make a set of dominos (i.e., include every possible domino). } \\
\text { How many distinguishable dominos would you make for a complete set? }\end{array}$ \\
\hline Language Books & $\begin{array}{l}\text { You have four different Russian books, five different French books, and six different } \\
\text { Spanish books on your desk. In how many ways can you take two of those books with } \\
\text { you, if the two books are not in the same language? }\end{array}$ \\
\hline CATTLE & $\begin{array}{l}\text { How many arrangements of the letters in the word CATTLE have the two T's appearing } \\
\text { together either at the beginning or the end of the word? }\end{array}$ \\
\hline $\begin{array}{l}\text { Basketball } \\
\text { Tournament }\end{array}$ & $\begin{array}{l}\text { A basketball tournament involving six teams is played each year. How many years must } \\
\text { the tournament be played to ensure that there is at least one repetition of a } \\
\text { championship matchup? }\end{array}$ \\
\hline Quiz Questions & $\begin{array}{l}\text { On a quiz, there are eight multiple choice questions, each of which has four possible } \\
\text { answers }(a, b, c \text {, and } d) \text {. In how many ways could a student complete this quiz? }\end{array}$ \\
\hline Cards & $\begin{array}{l}\text { In a standard 52-card deck there are four suits (hearts, diamonds, spades, and clubs), with } \\
13 \text { cards per suit. There are three face cards in each suit (Jack, Queen, and King). How } \\
\text { many ways are there to pick two different cards from a standard 52-card deck such that } \\
\text { the first card is a face card and the second card is a heart? }\end{array}$ \\
\hline $\begin{array}{l}\text { Increasing } \\
\text { Sequence }\end{array}$ & $\begin{array}{l}\text { You want to make a strictly increasing sequence of length three, using digits ranging from } \\
0 \text { to } 9 \text {. How many such sequences are there? }\end{array}$ \\
\hline Chords & $\begin{array}{l}\text { If you have } 10 \text { evenly spaced points around the perimeter of a circle, how many distinct } \\
\text { chords are there that connect two of the points? }\end{array}$ \\
\hline Security PIN & $\begin{array}{l}\text { A security PIN is a 4-digit sequence made of the numbers } 0 \text { through } 9 . \text { How many PINs } \\
\text { are there such that no two consecutive digits are the same number? }\end{array}$ \\
\hline Bob \& Jim & $\begin{array}{l}\text { In how many ways can seven people be seated in a line if Bob and Jim (two of the } \\
\text { people) do not sit next to each other? }\end{array}$ \\
\hline
\end{tabular}

permutations and combinations are fairly similar. More details about the reinvention are provided in the Results section.

Phase 3 (Sessions 8-10): Using the Formulas to Solve New Problems After the students had reinvented the formulas, we wanted to provide opportunities to apply them. During Phase 3, we were interested to learn whether the students would use the formulas they had just developed, and if so, whether the process of reinvention would support their facility in correctly applying them. We also wanted the chance to investigate any noteworthy aspects of their reasoning that emerged during work on any of the problems.

Table 3 includes the 15 tasks we selected for Phase 3. The first nine of these tasks reflect what we believed to be fairly straightforward instantiations of arrangements (with and without repetition) and combinations, and these were chosen to see if and how the students would apply the formulas they had reinvented. Although students might make unanticipated connections between problems, we nevertheless chose these problems according to which formula/solution type might most naturally fit them (from our perspective), providing at least two problems that could be appropriately answered with each formula the students had reinvented. The last six of these problems were 
Table 2 Phase 2 tasks - Reinventing counting formulas

\begin{tabular}{|c|c|c|}
\hline $\begin{array}{l}\text { Goal } \\
\text { formula }\end{array}$ & Problem Name & Problem Statement \\
\hline$n !$ & Library Books & $\begin{array}{l}\text { In the downtown public library, there are } 648 \text { books in the children's section. } \\
\text { In how many different ways can all of those children's books be arranged } \\
\text { on a shelf in the library? }\end{array}$ \\
\hline$n^{r}$ & Houses & $\begin{array}{l}\text { There are } 40 \text { houses in the neighborhood, and they each need to be painted } \\
\text { this summer. There are } 157 \text { paint colors available. In how many different } \\
\text { ways could all of the houses be painted? }\end{array}$ \\
\hline$\frac{n !}{(n-r) !}$ & Fan Prizes & $\begin{array}{l}\text { There are } 19,000 \text { fans at a basketball game. During halftime, a first, second, } \\
\text { and third place prize are going to be given out to three lucky fans. In how } \\
\text { many ways can the prizes be given out? } \\
\text { There are } 19,000 \text { fans at a basketball game. Throughout the game, } \\
50 \text { randomly chosen fans are going to be given } 50 \text { different prizes. } \\
\text { How many possibilities are there for how the prizes can be distributed? }\end{array}$ \\
\hline$\frac{n !}{(n-r) ! r !}$ & Fan Free Throws & $\begin{array}{l}\text { There are } 19,000 \text { fans at a basketball game. During halftime, three lucky fans } \\
\text { get to participate in a free throw contest. How many possibilities are there } \\
\text { for which fans can participate? } \\
\text { There are } 19,000 \text { fans at a basketball game. After the game, } 50 \text { fans are going } \\
\text { to be chosen randomly to meet the team. In how many ways can these } \\
50 \text { fans be chosen? }\end{array}$ \\
\hline
\end{tabular}

chosen and developed as Phase 3 unfolded, with the aim of investigating the students' reasoning about combinations. ${ }^{3}$

Analysis As the teaching experiment proceeded, we conducted an ongoing analysis that included reviewing the videotape of each session and constructing a content log of that session. In creating these content logs, we paid particular attention to the students' articulated thoughts that seemed to provide them with leverage, the voicing of concerns or perceived hurdles that needed to be overcome, and signs of/causes for progress. Our ongoing analysis informed our decisions about tasks for subsequent sessions. We also conducted a retrospective analysis (Cobb 2000) in which we reviewed the entire corpus of data at a deeper level, so as to refine our descriptions of thematic elements present in the students' reasoning. This retrospective analysis included re-watching all of the videos, enhancing the content log for each session, and noting recurring phenomena and themes. Once we had identified a handful of themes, we reviewed and transcribed key portions of the video in order to investigate aspects of the data related to each theme. Further discussions with the entire research team contributed to an appropriate framing of the findings within Lockwood's (2013) model.

\footnotetext{
${ }^{3}$ In Phase 3, the students seemed to have issues seeing the Bits problem as involving combinations. After they worked on the initial nine problems, we asked them six additional problems with the aim of better understanding their reasoning about combinations. We do not discuss these problems in this paper, but we include them for completeness and for consistency with other reports of this data (detailed further in Lockwood, Swinyard, and Caughman, in press).
} 
Table 3 Phase 3 tasks - Using the formulas to solve new problems

\begin{tabular}{|c|c|}
\hline Problem Name & Problem Statement \\
\hline SMOTHERING & How many ways are there to rearrange the letters in the word SMOTHERING? \\
\hline iPhones & $\begin{array}{l}\text { In a shipment of } 1000 \text { iPhones, } 25 \text { are defective. In how many ways can we select a set } \\
\text { of } 50 \text { non-defective iPhones? }\end{array}$ \\
\hline License Plates & $\begin{array}{l}\text { How many six-character license plates can be formed using upper-case letters and the } \\
\text { digits } 0 \text { to } 9 \text { ? }\end{array}$ \\
\hline Bits & $\begin{array}{l}\text { Consider binary strings that are } 256 \text { bits long. How many } 256 \text {-bit strings contain exactly } \\
75 \text { zeros? }\end{array}$ \\
\hline Horses & $\begin{array}{l}\text { Twenty horses are running in the Kentucky Derby. How many options are there for } \\
\text { which horses could finish with Win, Place, or Show? }\end{array}$ \\
\hline Coin Flips & A fair coin is flipped 36 times. How many outcomes have a head on the fifth toss? \\
\hline Lollipops & $\begin{array}{l}\text { There are eight children, and there are three identical lollipops to give to the children. } \\
\text { How many ways could the lollipops be distributed if no child can have more than one } \\
\text { lollipop? }\end{array}$ \\
\hline
\end{tabular}

Kickball There are 30 kids who want to play kickball in gym class, and in a game of kickball there are nine positions on the field at a time. How many ways are there for nine of these kids to play in a kickball game?

Paintings In the Portland Art Museum, there are 15,000 paintings. In how many ways can these pieces of art be displayed on the walls of the art museum?

Red Houses There are 40 houses in the neighborhood, 157 paint colors to choose from. I want to paint exactly two of the houses fire engine red. In how many ways can I paint all of the houses if exactly two of them must be fire engine red?

Red, White, Blue Now there are eight houses, 157 paint colors. We want 1 house to be red, 1 white, and 1 Houses blue, and the others can be any of the other colors. In how many ways can we paint the houses to accomplish this?

Flamingos There are 39 flamingos at the zoo. The zoo is going to exchange six of them with another zoo for five peacocks. How many possibilities are there for which flamingos are exchanged?

Blue Houses There are 40 houses on the block. In how many ways can three houses be painted blue?

Fans Revisited There are 40 fans at a Blazer game. In how many ways can three of them participate in a half-court shooting contest?

Fans in Houses $\quad$ Now suppose these 40 fans all lived on the same street in 40 different houses. We are going to pick 3 of the houses to paint blue. In how many ways can we do that?

The reader may note that some of the problems (Bits, Coin Flips, iPhones) have additional complexity. We felt that the students could still appropriately answer these problems by utilizing the formulas within their solution

\section{Results}

In this section, we describe the three phases of the teaching experiment, emphasizing that the students' use of sets of outcomes supported their successful reinvention of the four formulas and led to a high rate of success on subsequent problems.

Phase 1 (Sessions 1-4): Solving Initial Counting Problems In Phase 1, we presented the students with the ten initial problems listed in Table 1. The students' work in Phase 1 was marked by a focus on listing outcomes, and, in many cases, relying on patterns to determine the answer to each problem. As an example of their problem solving approach, we present their work on the Dominos problem. It is worth noting that the 
Dominos problem was their first exposure to solving counting problems together. We detail their work to highlight a couple of important norms that were established and note that their work on this problem is representative of their work with outcomes throughout the teaching experiment. Thomas began the Dominos problem by writing out a row of seven dominos, writing a 0 over a 0 to represent the 0:0 domino (Fig. 3). The students then had the following exchange, which set the stage for prioritizing listing over a search for an equation.

Robin: Isn't there an equation for that?

Thomas: I bet there is.

Robin: I don't remember it...Isn't it something like, what are those things called?

... Like combination equations... How should we do this?

Thomas: I don't know, I don't know the equation, so...(trails off).

After this exchange, the idea of simply looking for an equation was tacitly set aside. Without knowing what such a formula might look like, the students instead proceeded with listing more outcomes. Thomas said, "For each of the top half, there would be seven that match with it. So we'd have to do one for every one of them, 7, 7, 7, 7, 7, 7, 7 ," suggesting repeated addition. They then started to write a second row of seven dominos (1:0 through 1:6), but when they wrote 1:0 they quickly realized that they had already counted $0: 1$, and noted that they should not also count 1:0 because those two dominos should be considered the same. Thomas suggested that there thus would not be 49 dominos, but he had not yet discerned a correct pattern, saying, "so this one would have seven and then each of the rest would have like six." Robin then suggested that they write out more dominos, which they did.

As they wrote out more dominos, they listed the second row of dominos with $1 \mathrm{~s}$ and crossed off the duplicate 1:0 domino. They then began to write out the dominos with $2 \mathrm{~s}$ (the 3rd pair of rows in Fig. 3), and they crossed out 2:0, but as they wrote 2:1 Thomas said, "Ooooh, one more cancels each time." This suggests that the listing helped him recognize the duplication. They then wrote the entire list and crossed out all of the duplicates, finally computing the sum to find the correct total $7+6+5+4+3+2+1=28$.

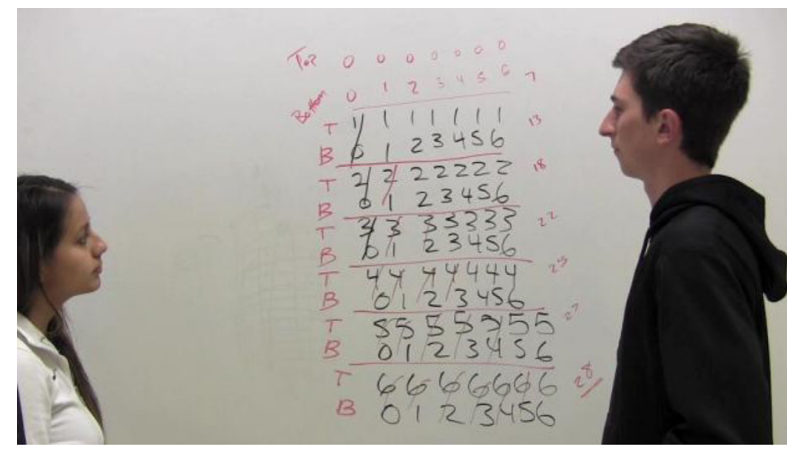

Fig. 3 Thomas and Robin's work on the Dominos problem 


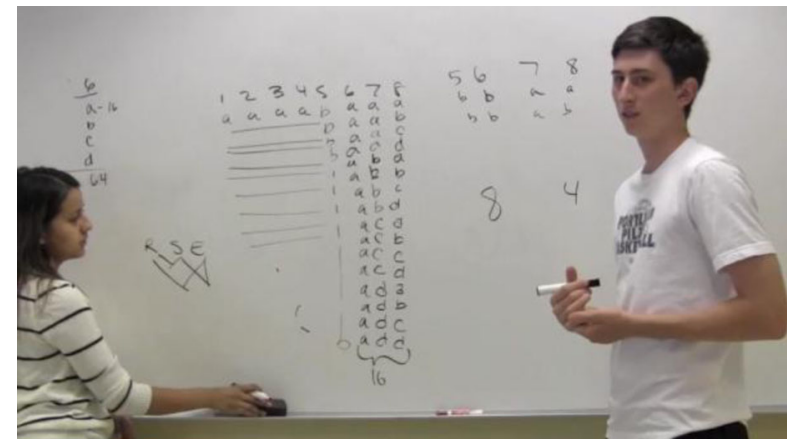

Fig. 4 The students' work on the Quiz Questions problem

This problem highlights the value of articulating and listing outcomes, particularly in recognizing issues involving overcounting. Indeed, it seems as though Thomas might not have recognized that "one more cancels each time" had he not listed more outcomes beyond the second row of dominos. Listing outcomes and seeing how overcounting occurred (and was avoided) gave them additional insight that simply writing a formula may not have provided. Because of this, we suggest that this problem was key in establishing a norm of valuing outcomes, and their work on this problem set the stage for much of Phase 1.

The Quiz Questions problem represents the one problem in the initial set that had a relatively large set of outcomes $(65,536)$ that certainly could not be listed by hand. Prior experience with this problem suggested that students typically reason using the multiplication principle (even informally) to solve it. Thomas and Robin created a table with eight columns for the questions 1 through 8 , and then tried to enumerate the possibilities by holding the letters in the first seven columns constant and then changing the last letter (Fig. 4). Accordingly, they wrote AAAAAAAA, AAAAAAAB, AAAAAAAC, and AAAAAAAD, and found there to be four options for changing the last letter. Even though they had the eight different questions labeled, at no point did they talk about the number of choices they had for each position, and they did not appear to think of constructing an outcome in stages by considering that there were four choices for each question. Rather, as we will see, they arrived at the correct answer of $4^{8}$ by noticing how quickly things were growing (suggesting exponentiation) and verifying this guess through establishing and checking a pattern.

After much initial work on the problem, including writing outcomes in a table, Thomas had written an 8 and a 4 on the board and had articulated his frustration about the magnitude of the answer. The excerpt below suggests that Thomas was motivated to pursue exponentiation because he realized the options were growing quickly.

Thomas: We're getting really frustrated trying to write out all of the possibilities, because we're just noticing it's just going to keep growing and growing. So we're trying to think of a way we can just either multiply them [referring to the numbers 4 and 8], or do something with them. I wonder, because it's growing really fast... I wonder if you would [writes an exponent of 4 on the 8 - they both laugh]. It's 
just, they're growing really fast, and so maybe exponentially, 'cause then that would give us a really large number.

After describing how the numbers were growing in a specific pattern, Thomas adjusted the conjecture and wrote $4^{8}$.

Thomas: Well because now, maybe it's not the 8 to a power, because we see that there's 4, changing just this last column, 'cause it's each one of the possibilities. And then when we move over a row, then we get like a total of 16 possibilities, which is 4 times 4 , or 4 squared, and so maybe if we go out, should we do this one [referring to the third to last column]? Oh we got 64 , which is 4 cubed, and so gosh.

Robin: What if it's 4 to the $n$ ?

Thomas: We've changed 3 questions and we've got 4 cubed. So if we changed the 4th question we'd hope to get 4 to the 4th. Ooh, my goodness [writes and calculates $64 \times 4=256$.

Robin: Yeah so what if it's just 4 to the $n$ ? I could see that.

Thomas: It's going to be hard to check it, because already we didn't want to count up like this fourth column, which we think would give us 256 , it's just going to be hard to check if we're right or not. It fits these first three. Um, I don't know.

The students' work on the Quiz Questions problem is representative of a method they consistently used to solve counting problems for which they could not easily list all of the outcomes. In particular, they would first write out some outcomes and formulate a pattern, perhaps for a smaller case. Then, if they became overwhelmed by the magnitude of the answer, they would explore an operation that might give a sufficiently big answer. That is, they would operate with the numbers in the problem to see if they could yield numbers that matched with the pattern they had established. In the Quiz Questions problem this operation was exponentiation, but at other times they multiplied numbers or used factorials. As we will see, they continued to employ this method throughout the other phases of the teaching experiment.

The Bob \& Jim problem (Table 1) serves as another example during Phase 1 in which the students reasoned with outcomes but did not list the entire set of outcomes. The students articulated what they were trying to count, eventually encoding an outcome as a sequence of B, J, and the numbers 1 through 5 (representing Bob, Jim, and the other five people), where B and J could not be next to each other. They began by writing $\mathrm{B} 12345 \mathrm{~J}$, then $1 \mathrm{~B} 234 \mathrm{~J} 5$, then $12 \mathrm{~B} 3 \mathrm{~J} 45$, as possibilities, but then they realized that the placement of Bob and Jim did not need to be symmetric. They counted (through complete listing) the 15 ways in which Bob and Jim might be placed nonconsecutively in two of the seven locations. They also recognized that Bob and Jim could switch places in any of those 15 possible arrangements, so they multiplied 15 by 2 to get 30 possible ways in which Bob and Jim could be seated non-consecutively. They then realized that they needed to figure out how to 
arrange the remaining five people, and they noted that whatever that number is, they should multiply it by 30 .

What we want to emphasize about this problem, though, is their work when they tried to determine how many ways there are to arrange five people. While a natural approach may be to use the multiplication principle, and to think of five choices for which a person could be in the first position, then four remaining choices for the second position, then three for the third, two for the fourth, and one for the fifth, the students did not solve the problem in this way. Instead, consistent with their prior work on the Quiz Questions problem, they empirically determined that for 1, 2, 3, and 4 objects there were 1, 2, 6, and 24 arrangements, respectively. When trying to think of how to arrange five people, they used the patterns they had established. Here, Thomas noted that it's "doubling" then "tripling" and "quadrupling," and so he suspected that maybe they should multiply by five to continue the pattern. Their reasoning about the arrangements of five people was based heavily in pattern recognition, and they relied on their empirical work with a series of small cases in computing the arrangements of five objects. The students ultimately were able to solve the problem by multiplying 30 by 120 to find the final correct answer. It is noteworthy to us, however, that 120 was built up by a series of patterns and did not seem to be grounded in reasoning using the multiplication principle. Further, despite the fact that they multiplied the results of the two stages of the problem (arranging Bob and Jim and then arranging the remaining five people), they never verbalized reasoning suggestive of a rich understanding of the multiplication principle. Thus, although they multiplied, and although they demonstrated perhaps some sense of the multiplication principle on this problem, we do not have evidence that they possessed a rich understanding of the multiplication principle or that it was a well-developed tool for them.

Summary of Phase 1 Thomas and Robin correctly solved all ten problems they encountered during Phase 1, which is noteworthy in and of itself. Their work on the first ten problems established norms for what constituted an acceptable answer and how they would approach problems. They regularly focused on sets of outcomes, identifying what they were trying to count and using their listed outcomes to avoid counting duplicates. We also observed them employing a consistent method for problems involving large sets of outcomes - they regularly used empirical patterns to solve such problems.

Phase 2: Reinventing Counting Formulas In Phase 2, we sought to have the students reinvent the four basic formulas. Our main strategy was to give them problems with large sets of outcomes, so they would be motivated to generalize a strategy and ultimately a formula. We conjectured that the students might formulate smaller problems first, and perhaps develop patterns or discern relationships that they could use to derive the formulas. We hoped that engaging them in this process would both help them arrive at the formulas and support them in making sense of why those formulas work. Indeed, our overall motivation was that by coming up with the formulas themselves, instead of being directly told or taught them, the formulas might be more meaningful for the students.

Through engaging with the problems, the students successfully reinvented each formula. Table 2 shows the problems designed to facilitate the reinvention of each respective formula, and Table 4 compares common textbook formulas with the 
expressions reinvented by the students. The students used notation that we interpret as being meaningful for them, based on their experience - for instance, the $f$ in the last two formulas stood for fans at the basketball game. In this section we describe their reinvention of each of the four formulas.

Reinventing the Formula $n$ ! for Permuting $n$ Objects To facilitate the reinvention of this formula, we gave the students the Library Books problem (Table 2), which involves arranging 648 books on a shelf. The students began by building on their prior work on the Bob \& Jim problem, in which they had determined that there were 120 ways in which to arrange five objects. In the Library Books problem, they immediately realized that the solution would be extremely large, and they would not simply be able to exhaustively list the outcomes. They repeatedly mentioned feeling overwhelmed by the magnitude of the set of outcomes.

Robin: Well, we can get a system going, just to see patterns, maybe?

Thomas: 648, though? Um, kind of big... 'Cause you can literally move one book, and it'll be completely different.

In their work on the Bob \& Jim problem, which came directly before this Library Books problem, recall that the students had thought of the multiplication in that problem as building up, and, as they put it, as "doubling, tripling, quadrupling" each time. They had felt that any multiplication they did had relied on the previous answer being known - they knew the answer was 120 because they had found 24 (the number of ways of arranging four objects) empirically, and they reasoned that they had five groups of 24. The building up had not been an issue for them in that problem, because they only needed five steps, which they easily computed. They even went on to reason that for six books it would be six groups of 120, or 720 total. In the Library Books problem, though, while they knew that they could find the answer by multiplying the answer for 647 paintings by 648, they thought that they could not determine their final answer without multiplying in 648 steps. Feeling overwhelmed by this and realizing they could not realistically complete 648 steps, the students (in line with their method described previously) then tried to manipulate numbers to match their pattern. They felt confident that there were 120 ways to arrange five objects, and 720 ways to arrange six objects, and so they started to perform operations with the number 5 to try to yield 120 , and with the number 6 to yield 720 . We infer that at this point they had limited operations - multiplication and exponentiation - at their disposal, and, based on the pattern they had observed, they were trying to see which technique might give them the answers they knew were true. Evidence for this is seen in the excerpt below.

Table 4 Textbook formulas and students' reinvented formulas

\begin{tabular}{lllll}
\hline Textbook formulas & $n !$ & $n^{r}$ & $\frac{n !}{(n-r) !}$ & $\frac{n !}{(n-r) ! r !}$ \\
\hline Students' formulas & $n !$ & $b^{a}$ & $n ! \div(n-f) !$ & $\frac{n ! \div(n-f) !}{f !}$ \\
\hline
\end{tabular}


Robin: We don't really know how to approach it, so we're going back to what we did with the previous one, where we could switch up, you know the 5, what was it, ABC, I can't remember.

Thomas: It was like numbers of people.

Robin: Numbers of people, and so we found that we can switch five 120 ways, but we have to figure out [points to 648].

Thomas: 648 is really big, and we can't - before we'd do a table and try to do a couple options to find a pattern, but it's too big. And so we went back to smaller numbers that we knew how to switch up, and we're trying to find...we found a basic pattern of like it'd double then triple then quadruple, and keep going, but then we're at 6 [points to 6], and to get to 648 would just be a lot. It'd just be a lot to keep going one by one, and so we're hopefully trying to find another pattern that's connecting these that we can apply directly to 648 .

Int. ${ }^{4}$ : What do you mean by directly to 648 ?

Thomas: So we don't have to rely on like 647, that number, and then times that by something to get the 648th one.

Int.: Oh I see, so you can go like directly to 648 .

Thomas: Mm-hmm.

Int:: I see, okay.

Thomas: And so we're just trying to play around with like taking them to a power or something, and it's not working.

Robin: Yeah, not working.

Next, Robin suggested that perhaps there are other "things" they could do to operate with the numbers, and Thomas wondered if the operation might be factorial. In the excerpt below they wrote down descending products of $5 \times 4 \times 3 \times 2 \times 1$, then $4 \times 3 \times 2 \times 1$, and arrived at the correct answers of 120 and 24, respectively.

Robin: Can't we do other things, like, kind of like this [points to exponents], but, um...

Thomas: What if it's [and writes $5 \times 4 \times 3 \times 2 \times 1$, and multiplies out 20, 60, 120]. Hmm [surprised sound].

Robin: Factorial?

\footnotetext{
${ }^{4}$ Int. stands for interviewer.
} 
Thomas: Maybe [then writes $4 \times 3 \times 2 \times 1$ and multiplies out to get 24]. Yeah, that's looking good. 'Cause 3 is the 6 .

Robin: What's 6 ? 6 times 120. Ahhhh.

Thomas: So we're thinking the pattern may be factorials. So far with this pattern, however many numbers we have to mix up, you just do the factorial of it, it gives you that number.

Robin: Yeah.

Thomas: And so I guess right now we'd say that our answer would be [writes 648!] something massive.

The explanation below gives further evidence that the students were employing their method of trying to operate with numbers to fit a pattern. It also suggests that Thomas' decision to try factorial was somewhat serendipitous.

Int.: And what made you decide to just write that ...?

Thomas: Well she just kind of brought up the idea, 'cause we were just throwing out anything, she was maybe it could be something else, and so I was just like, well, maybe, and we kind of just went on a limb and...

Robin: It happened to work out.

They were then asked what the answer would be for arranging any number of objects, and they correctly concluded that there would be $n$ ! ways to arrange $n$ objects. Although we note that their reinvention was heavily based on numerical patterns that they noticed in building up from smaller problems (some of which had been solved in previous problems, such as the Bob \& Jim problem), the overall strategy of having the students work with bigger numbers so they would be motivated to generalize did seem to facilitate their correct reinvention of the formula.

Reinventing the Formula $n^{r}$ for Arrangements with Repetition For this formula, we gave the students the Houses problem (see Table 2). Here Thomas recognized almost immediately that this was like the "multiple choice" problem (the Quiz Questions problem), and he had written out the numbers 8 and 4, and then below them the numbers 40 and 157 (see Fig. 5).

The following discussion demonstrates how they drew on the similarity between the Houses problem and Quiz Questions problem to come up with their correct answer.

Thomas: This is a lot like the multiple choice one.

Robin: Yeah. 


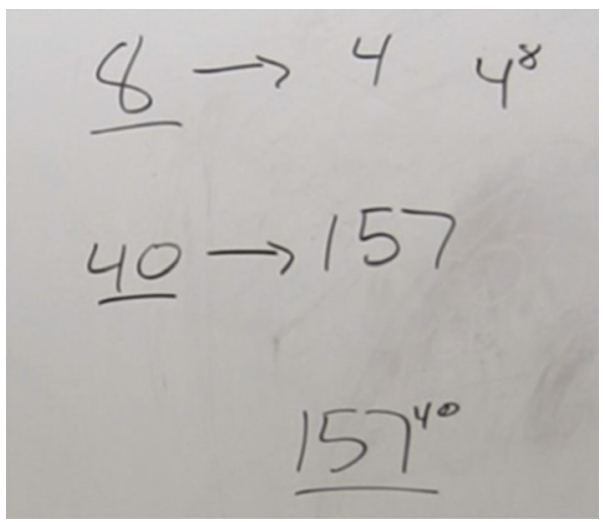

Fig. 5 Comparing the Houses problem to the Quiz Questions problem

Thomas: Because, there was, the multiple choice one was ... There was like 8 questions just like there's 40 houses. Each question has 4 possibilities, just like each house has 157 possibilities.

Robin: So $157 \ldots$ trails off as Thomas writes $157^{40}$ ].

In establishing the similarities between the Houses and Quiz Questions problems, the students recognized that the outcomes being counted in both problems are similar sequences in which order matters and repetition is allowed. For example, Thomas said that one outcome would be to paint all of the houses red, and Robin clarified that repetition was allowed by making sure that Red, Red, Yellow and Red, Red, Blue would be allowable outcomes. Thomas was asked to summarize his work, and as further evidence of their thinking on the problem, Fig. 5 shows the clear analog that he saw between the two problems, as well as how he used the previous experience on the Quiz Questions problem in determining an answer for the Houses problem.

Thomas: So right away I think we both thought of the multiple choice problem from earlier...Right off the top of my head I'd got to $157^{40}$, because in the previous problem it was $4^{8}$, and we did like number of possibilities per thing to the power of how many we had, which would be that [refers to $157^{40}$ ].

From here, it was not difficult for the students to generalize to a formula. We asked them in general to come up with an equation for, using their language, "filling a certain number of things with a certain number of options." Thomas' response shows how they arrived at their version of the reinvented formula.

Thomas: If $a$ is your things you're going to fill, so like a bucket or something like that, and then $b$ is just what you could fill it with ... different liquids for the bucket. We would I think just go, $b^{a}$. Like your possibilities of filling to the power of how many things need to be filled.

The students thus correctly reinvented the formula, drawing upon their prior experience on the Quiz Questions and Houses problems to do so. 


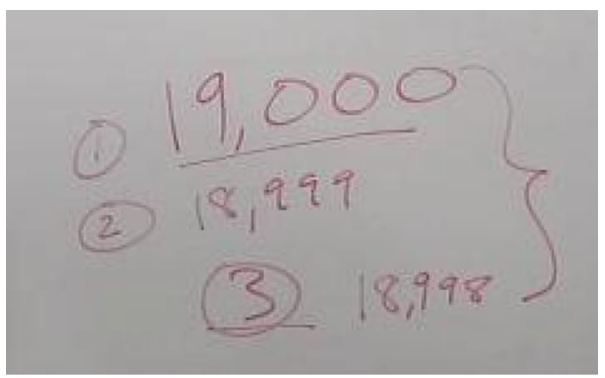

Fig. 6 The students' work to reinvent the formula for permutations

Reinventing the Formula $\frac{n !}{(n-r) !}$ for Arrangement of $r$ Objects from $n$ Distinct Objects To encourage the students to reinvent the formula for arranging $r$ objects from $n$ distinct objects, we proceeded in two stages, again adopting the premise that providing situations with larger answers would motivate the students to be more general in their thinking. To do this, we first gave Thomas and Robin the Fan Prizes problem (Table 2), involving 19,000 fans getting three prizes, where one number was large $(19,000)$ but the other (3) may not require generalization. We then gave them another problem involving 19,000 fans and 50 prizes, increasing the second number to motivate generalization. We thus gave two subsequent problems to foster this reinvention.

Thomas and Robin proceeded much in the same way as before, drawing on previous problems and relying heavily on patterns that they had observed. By considering an outcome, they determined that they were in a novel situation and could not solve the problem using the formulas they had already established.

Thomas: You have 19,000, and then for the second prize you only have that many [writes 18,999], and then the third prize [writes 18,998]. But then it doesn't keep going... it's just like these three [writes a bracket around the three numbers (see Fig. 6)].

Initially they could not decide what to do with these three numbers and decided to explore a smaller problem. At first they figured out the number of ways to arrange three numbers from four (instead of three from 19,000), and they found that there were still 4 ! ways to do so. ${ }^{5}$ Thus, they conjectured (incorrectly) that because there was not a difference between a smaller factorial and the original factorial in that smaller case, the answer to the original problem would just be 19,000 !.

Thomas: If there's 19,000 people total, that's how many people who could win the first prize. But once they pick the first prize person, then that person can't win again. So then there's one less to win second, and one less to win third, and that looks like, to me at least, when we were doing the books, or even any other factorial problems. Where it'd be like, I forgot what the numbers are but you've got like 1,000 books for the first possibility, and then one less for the next, and one less for the next. But with those we went all the way down to one, which looked like a factorial, but this one we're only going down the first three, which is

\footnotetext{
${ }^{5}$ It happens to be the case that permuting four objects is the same as permuting three from four objects.
} 
why I kind of want to look at a scaled down version because I'm not sure if it's just these three numbers multiplied together [referring to the 19,000, 18,999, and $18,998]$, or if it's something a little different.

However, Thomas then recognized that he had chosen a special example (where permuting three of four objects is the same as permuting all four objects), and so they began writing out all 12 ways of arranging two objects from four. Next, Robin suggested that they do an additional smaller problem by arranging three out of five objects, and she began listing such arrangements, where 1 is the first number. They found 12 such possibilities, and they recognized that for any of those 12 possibilities they could pair them with any of the five numbers, and they thus found 60 ways to permute three from five objects. We interpret that they used repeated addition here, seeing five groups of 12 . Then, Thomas wrote the numbers 5, 4, and 3, mirroring what he had written for the 19,000 problem (see Fig. 7), suggesting that he was drawing on the smaller case that they had written out in order to make connections with the current problem. He then quickly wrote out his smaller case of arranging two from four people, noting that there were 12 possibilities in such a case (Fig. 7), and he then pointed to the bracketed three numbers written on the board in Fig. 6 and said "this is looking pretty good." Thomas and Robin had thus established a pattern in their smaller cases of $4 \times 3$, and $5 \times 4 \times 3$, and this led them to say that perhaps the answer is the product $19,000 \times$ $18,999 \times 18,998$. They viewed the smaller cases as more of a check than providing actual insight into their answer. Here we highlight that while they ended up at the correct product, their work was consistent with prior problems in which they empirically derived patterns and used them to argue for a particular answer.

We then asked them to solve a variation of the previous problem, in which 50 fans are chosen instead of three. They immediately connected the new problem to the previous problem. They recognized that they wanted the product of 50 terms starting with 19,000 and continuing to 18,951 . It is noteworthy that the larger number (50) did motivate them to find a shorter way to write the number instead of writing out the whole product, but they initially (incorrectly) wrote 19,000! - 18,950!, using subtraction instead of division. In an effort to understand why they had chosen to use subtraction, we asked them to consider a smaller problem. As they considered a smaller case, they recognized their mistake. In particular, they went back to the previous work they had done to determine the number of ways to arrange three objects from five objects (60). They noted that 5 ! -2 ! would give them $120-2=118$, which is incorrect, so they realized they needed to try something else. In explaining their work, Robin said, "So then we thought, well how do we get 60 using the last 2 numbers [points to the numbers 1 and 2], and we divide by 2." Thus, by considering smaller problems more carefully, and specifically by manipulating numbers and checking their work, they realized that they should use division instead of subtraction. They eventually recognized that they could use the factorial notation as a shorter way to write the desirable products. After some work, then, the students wrote out their own formula for how many ways to arrange $a$ objects from $n$ objects (Fig. 8). We suggest that their use of the division symbol instead of the horizontal division bar was based on how they had initially written the subtraction. When asked what the correct answer would be, Thomas simply added a dot above and below the subtraction sign (Fig. 9a and b), and that notation persisted for the students as they continued in the teaching experiment. 


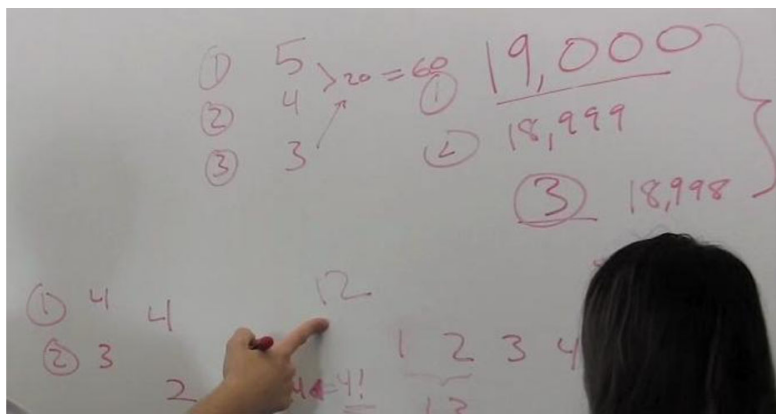

Fig. 7 The students look for patterns using smaller cases

Reinventing the Formula $\frac{n !}{(n-r) ! r !}$ for Selecting r Objects from $n$ Distinct Objects To help Thomas and Robin reinvent the formula for selecting objects, we gave them the Fan Free Throws problems (Table 2) that involved fans participating in a half-time contest. These tasks were similar to the previous problems that asked about giving prizes to fans, but here there was no ranking among the fans who participated. In reinventing the formula for combinations, the students again followed the same general method of their prior reinventions. First, they seemed to understand that the outcomes they were counting were different than in previous problems, which they demonstrated by writing some small sample outcomes in order to justify what they were doing. For example, Thomas indicated that "maybe it's different" from the previous problem, saying, "These fans aren't ranked. If there's fan a, b, and c to participate, then that's the same as fan c, b, and a to participate." He also recognized they should not use exponentiation, saying, "it's not the exponents because we can't have the same guy do it every time [writes $a, a, a]$." The excerpt below underscores this.

Thomas: What I'm thinking is that since these aren't like prizes, or they're not like in any order, these two combinations [writes 123, 321] I think would be counted as the same, because if you were to bring people in for a contest, it's still the same three people participating in a contest...Like the order in which the people are called or something doesn't matter.

Robin: Uh-huh. Yeah. It's just that they can't be called again.

Thomas: Mm-hmm.

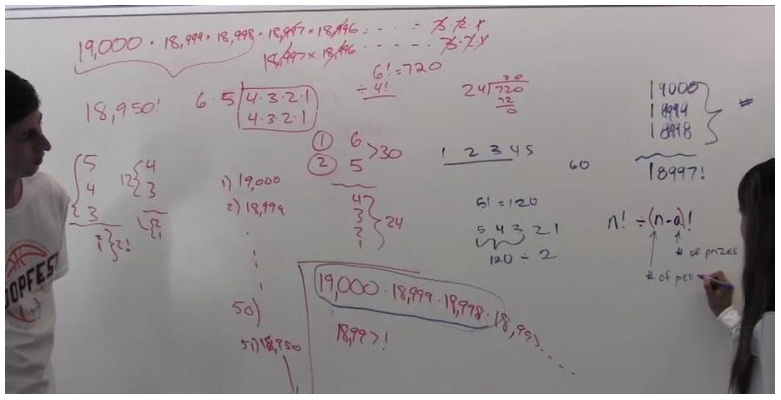

Fig. 8 Reinventing permutations of $a$ objects from $n$ distinct objects 
(a)

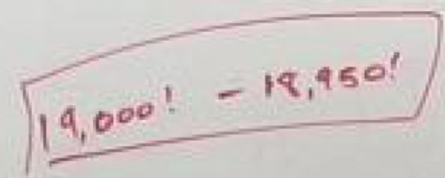

(b)

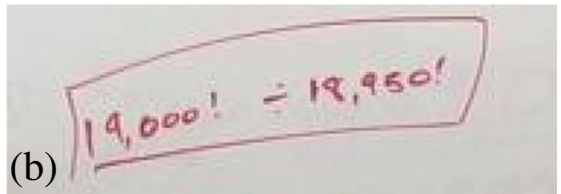

Fig. 9 a Students' initial incorrect response. b Students' corrected formula

The students then proceeded to spend time listing possible outcomes, empirically determining patterns and relationships. When we asked what they were doing, Robin told us that they are "looking for a pattern," which is in line with much of their previous work. When their pattern hunting proved fruitless, we intervened and suggested that they consider the differences between this problem and the previous problem. Thomas responded by listing out all six ways of arranging three letters, and recognized that all of those outcomes would only get counted once in the new situation. Hence, they recognized that while there are six distinct ways to arrange three objects, these six outcomes comprise a single "group" (see Fig. 10). Throughout the rest of the teaching experiment, Thomas and Robin consistently used "group" language to refer to a set, rather than an arrangement, of objects.

Figure 10 shows the ratios they had written, showing the arrangements on the left and how they correspond simply to a single group. They had written the group on the bottom of Fig. 10 involving arranging three objects (yielding a 6:1 ratio) first, and then they also tried an even smaller case of arranging two rather than three objects and found a 2:1 ratio.

After more discussion, they came up with the idea that there is a ratio of $6: 1$ between arranging and selecting objects (in this case, fans for participation), and they concluded that the answer to this problem should be the answer to the previous problem divided by 6 . Thomas then wrote $\frac{19,000 \cdot 18,999 \cdot 18,998}{6}$ on the board, but he said, "but why?" right after he wrote it. This suggests that while they had empirically realized the ratio of $6: 1$, they had not yet recognized why that relationship held (in particular, they did not yet

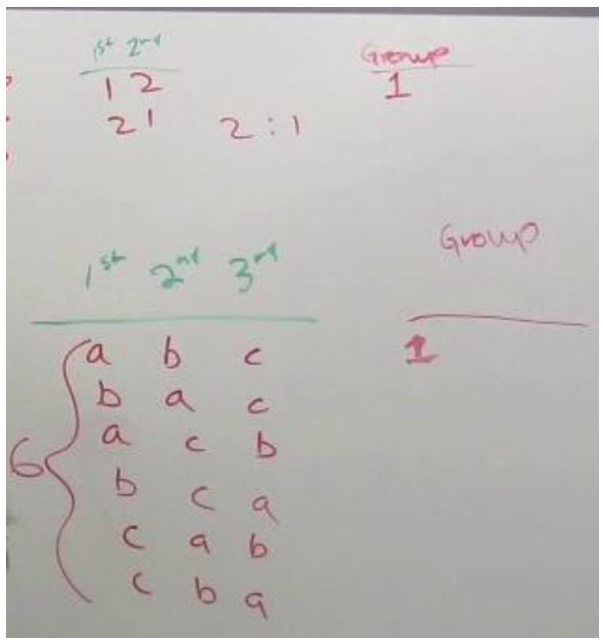

Fig. 10 Students examine ratios in smaller cases 
seem to realize that it was 6 because there are $6=3$ ! ways to arrange three people/ objects). In exploring this, Thomas had an 'aha' moment, exclaiming "oh my goodness!" when discovering that the ratio held. It seems that while they recognized that the pattern of dividing by 6 held, they were legitimately surprised as to why division by that particular number worked. When they needed to be more general they did see that $6=3$ !, but they recognized that because they had empirically determined that 3 ! happened to equal 6 . They did not express any recognition of 6 as a product of $3 \times$ $2 \times 1$.

The students then moved to the problem involving selecting 50 fans, which again pushed them toward generalization (the writing that corresponds with the excerpt is in Fig. 11).

Thomas: So for the first one we kind of found, kind of like 3, because there's three people going out...so this one would be like 50 [writes 50], and so for the prizes, how many combinations were there to one group, kinda? That ratio would help us, I think. Do you get what I'm saying?

Robin: Kind of, keep going.

Thomas: 'Cause for the first one, for three people [writes 3 on the board] with the prizes there were six combinations [writes an arrow from the 3 to 6] to one group [writes an arrow from the 3 to 1 ].

Robin: Uh-huh, oh oh oh oh.

Thomas: If you think of it that way. So for 50 [has written 50 on the board] it'd be something to one group [writes an arrow from 50 to 1], and then with that ratio we could use the answer we found in the second problem. Is it just this? [writes an arrow from 50 to 50 !], because this is...

Robin: 3 factorial?

Thomas: [Writes an $=3$ ! next to the 6] Because that was that.

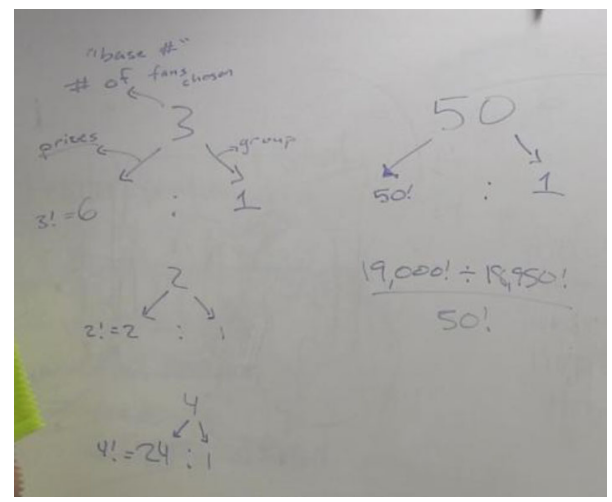

Fig. 11 Using and explaining ratios in arguing for a formula 
Based on the pattern of ratios Thomas said, "And so it could be [writes $\left.\frac{19,000 ! \div 18,950 !}{50 !}\right]$ by this reasoning [pointing to ratios he'd written on the board]." They checked one more example and labeled one of their diagrams (Fig. 11), explaining what they had found.

We see that they arrived at the correct answer, and that they drew heavily on the previous problem and the ratios that they had determined in their exploration. We note, though, that when asked to explain why division by 50 ! makes sense, their explanations were based exclusively on the pattern they had recognized. They knew to divide by 50 ! because they knew empirically, through patterns, that factorial was appropriate here. For instance, Robin's following explanation of their work suggests that they used the pattern of the ratios they had found.

Robin: We found this little triangle pattern, that if three are chosen for the activity where you can't do first, second or third, we found that it had six options. And what he discovered is the 6 came from, or could be related to 3 !, which was the base number, the number of people chosen, and so we saw that it worked for 2 as well, and we had this little ratio thing because, um, in the first problem there's six different options, but in the third problem there was, for every six there was one, and so that's why he has the ratio thing going, and he checked it for 4 just to make sure, and it seems to be right, so we did the same thing for 50 .

Then, when asked to solve a more general statement of the problem, they said it would be $\frac{n ! \div(n-f) !}{f !}$, and Thomas wrote that $n$ is the number of fans at game, and $f$ is the number of fans chosen for free throws (Fig. 12). The way in which they wrote the combination formula reflects that they derived it from (or that it is closely related to) the permutation formula. We note that the fact that they used unconventional notation suggests that the formula was meaningful for them.

Summary of Phase 2 By the end of Phase 2, the students had successfully reinvented four basic counting formulas, using their own notation. As researchers, we had made two design decisions with the hope of fostering that reinvention - providing problems with large numbers that would motivate generalization, and providing similar contexts for permutations and combinations to allow the students to capitalize on natural relationships between those two situations/formulas. We feel the results show that these decisions were effective, as the students were motivated to generalize on problems with larger sets of outcomes, and the reinvention of combinations was very closely tied to their work on permutations. In Phase 2, the students maintained their practice of regularly looking at sets of outcomes, being careful and precise in their work. We also saw the continuation of their consistent method for their work on problems involving outcomes that were too numerous to list completely. Indeed, their reinvention of all four formulas tended to be primarily based on empirical listing of outcomes, which they used to detect patterns that would help them generalize the formulas. We should also note that they solved all of the problems correctly in this phase.

Phase 3: Using the Formulas to Solve New Problems In Phase 3, the students were initially given nine new problems, as we wanted to see whether (and if so, how effectively) they would use the formulas they had just reinvented. What is most 


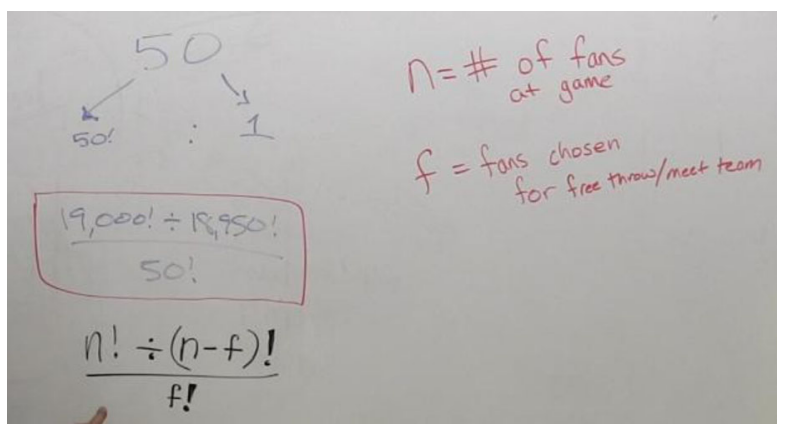

Fig. 12 The students' final combination formula

noteworthy in this phase is that even when armed with their new formulas, the students did not blindly or carelessly apply them. Instead, they articulated what an outcome was, discussed the nature of that outcome (whether repetition was allowed and whether order was relevant), and then used that discussion to determine which formula to use. In this way, they solved eight of these nine problems correctly. The one problem they did not answer correctly was the Bits problem, which they did not view as a problem involving combinations. Their work on the Bits problem prompted us to ask six additional problems at the end of Phase 3. Although we do not discuss this problem in detail in this paper (we discuss it further in Lockwood, et al., (in press)), we note that the students did not appear to understand that they could use combinations as a stage in the counting process, a fact which further indicates a somewhat narrow facility with the multiplication principle.

As two examples of the students' work in Phase 3, Figs. 13 and 14 show their (correct) work on the Kickball and License Plates problems (see Table 3), respectively. Figure 13 shows how they first wrote down an outcome and decided that different orderings of kids yielded different outcomes, which suggested permutations rather than combinations. They subsequently chose the appropriate formula and solved the problem correctly. Again these examples demonstrate how their facility with outcomes positively affected their work, helping them decide which formula to apply in which situation. Likewise, for the License Plates problem (Fig. 14) the students' use of outcomes helped them determine the nature of what they were trying to count, which prompted them to use the correct formula (in this case, their $b^{a}$ formula).

As a final example of their work in this phase, we detail their work on the Coin Flips problem, with the purpose of showing that their problem solving method from earlier phases of the teaching experiment persisted even to Phase 3. The students first wrote down pertinent information from the task, writing the numbers 1 through 36 and writing $\mathrm{T} / \mathrm{H}$ to represent tails or heads options for the first several coin flips. They then circled 5 as having to be a head (Fig. 15). They said that the problem reminded them of the Quiz Questions problem, and they quickly recognized that without the extra constraint of the 5th flip having to be a head, the answer would be $2^{36}$. Thomas said, "There's like 36 questions in a way, and like two answers, so it's like, but then I don't know about that [pointing to the 5th flip being a head]". He made a guess about what would happen if they did consider the 5th head constraint, arguing, "What if we kind of like took this out and said there was only 35 flips, because this one is always going to be heads, but then would that mix up the rest and then leave that one as heads [points to 5]?" He 


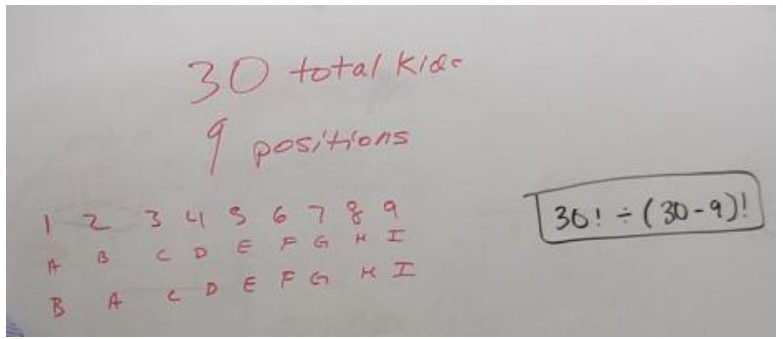

Fig. 13 The students' answer to the Kickball problem

thus wrote down a guess for the problem, but they were not yet sure of the answer. Robin recommended, "We could scale it down," and Thomas agreed saying, "I think the scalingdown way would be good, just to try to, like, test it out."

In order to check whether their $2^{35}$ answer made sense, they went to a smaller case and wrote down outcomes. Figures $16 \mathrm{a}$ and $\mathrm{b}$ show that they tried to solve the smaller problem of five flips in which the third flip must be a head. Robin wrote down every such outcome, as they together made sure they had all of the possibilities. She then wrote the numbers $1-16$ to the right of the outcomes (see right side of Fig. 16a), suggesting they determined that there were 16 such possibilities by physically counting up how many there were. Thomas' explanation provides further evidence that they based their work on previous problems and on empirically checking the smaller case. There were 16 possibilities because they listed out and counted 16 outcomes, but not because they reasoned that the answer was $2 \times 2 \times 1 \times 2 \times 2$ by considering the number of options for each flip, as the multiplication principle would suggest.

Thomas: So there's 16 with five. And if we were to do $2^{5}$ would give us 32 [writes $2^{5}=32$ ]...Alright and then $2^{4}$, because if we took this one out would give us 16 , which would give us that.

Robin: Ahh.

Thomas: Looking good. [To the interviewers, to explain his and Robin's work.] We're going to go with $2^{35}$, because we thought of pretty early that this looks like the multiple choice problem and like the housing problem where it'd be

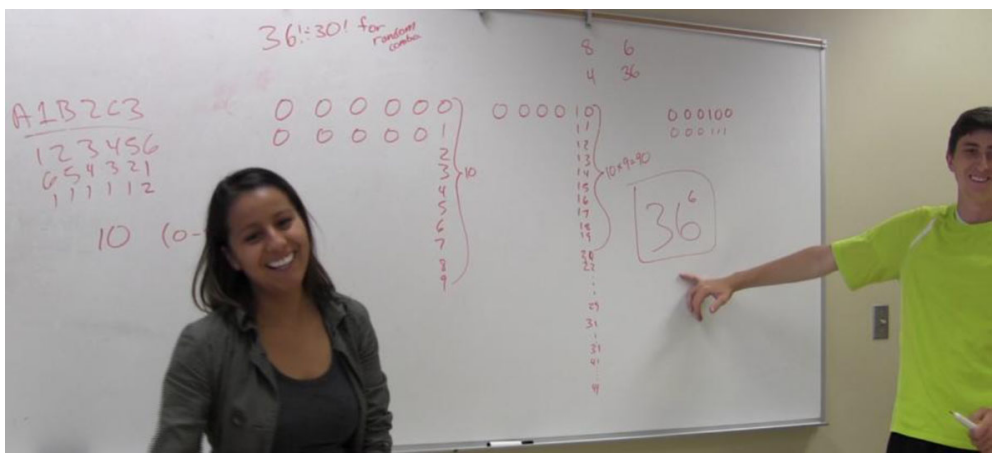

Fig. 14 The students' answer to the License Plates problem 


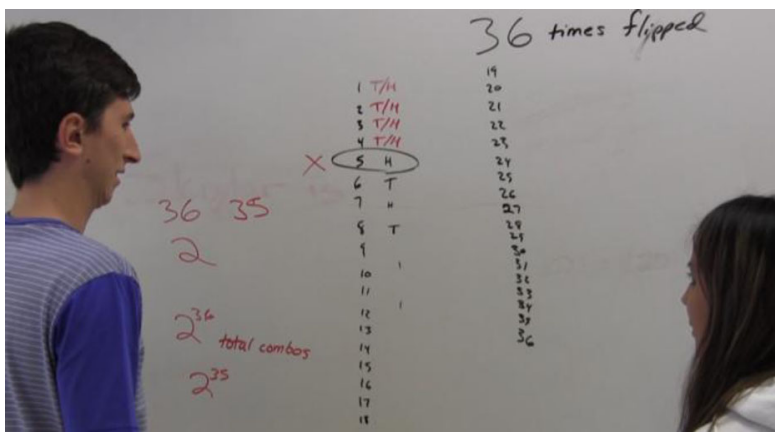

Fig. 15 The students' work on the Coin Flips problem

something to a power, and 36 was like our number of questions, and two was like our possible answers, but then the problem was how would we account for the 5 th one always staying heads? And so we scaled it down with only five flips with the third one always being heads, and we got 16 possible questions and combinations. And then we thought of, we could take the 5 th one or the 3 rd one there that always stayed constant out, reducing the number of flips by one, and then, so raising two to that number, which would be 35 here [pointing to the original problem] or four in this example [pointing to the smaller problem] and seeing if we got the right answer. And we checked it with the smaller numbers [pointing to the 16 they had written out] and concluded that the big version would be the same.
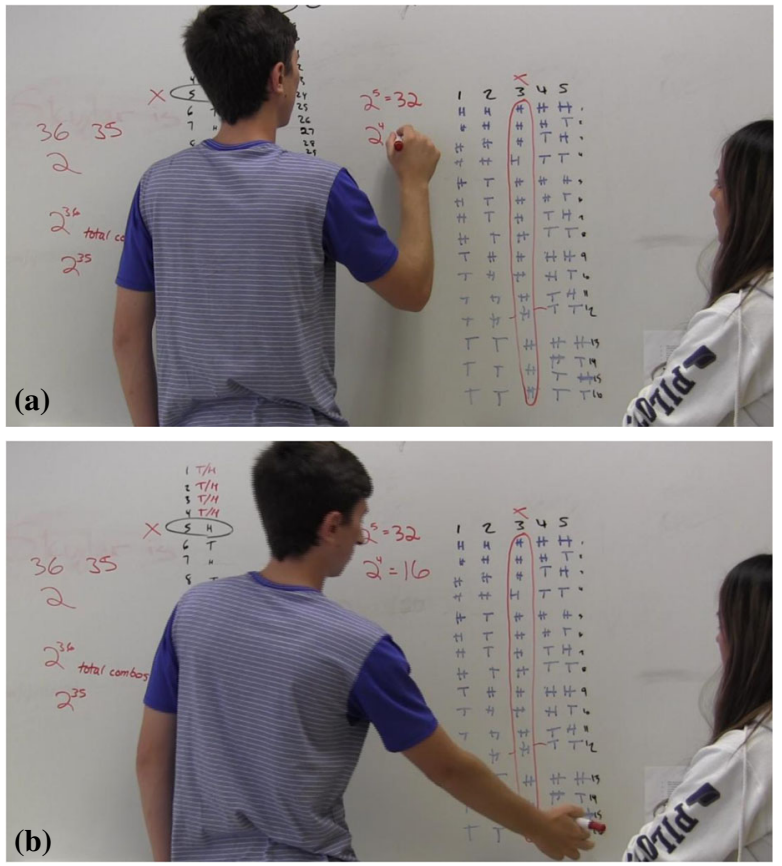

Fig. 16 a Checking the equation for a smaller case. b Empirically verifying the smaller case 
Thomas' language confirms that their work on this problem was empirically based. We want to emphasize that even in this third phase, when they had access to formulas they had previously constructed, their work was consistently grounded in sets of outcomes and empirical patterning.

Summary of Phase 3 In Phase 3, the students put the formulas they had just reinvented to use. It is important to note that the students did not abandon their work with sets of outcomes, and they did not blindly apply these formulas without reasoning about which formula might make sense. Rather, their work was once again characterized by first determining the nature of an outcome - of what was being counted in the problem and then deciding which formula appropriately counted that kind of outcome. We also saw that the students were consistent in their use of patterns and in matching up previous problems, as was evidenced in the Coin Flips problem. By adopting this method on a set of new problems, the students again saw considerable success solving eight of the nine new problems correctly.

Summary of Results We want to emphasize three aspects of the teaching experiment. First, throughout their work on these problems, the students used sets of outcomes frequently and with great effect. As discussed in their work on the Dominos problem, the students reasoned about outcomes to a much greater extent than demonstrated by students in previous studies (e.g., Lockwood 2011a). Second, the students used such reasoning to successfully reinvent the formulas, and, even more, they went on to solve problems correctly using these formulas. Again, we judge this to be a notable finding, demonstrating both that students are capable of reinventing counting formulas, and that they are able to use those formulas successfully in subsequent counting activity. Finally, the students were remarkably successful at solving these problems. Over the entire course of the teaching experiment, they solved 30/31 (97\%) of the problems correctly. We cannot overstate how noteworthy and impressive these results are, given the low success rates and difficulties that have frequently been reported in other studies (e.g., Eizenberg and Zaslavsky 2004; Lockwood 2011a, Lockwood and Gibson 2014).

\section{Discussion}

The findings above highlight two students' successful reinvention of four basic counting formulas, demonstrating facility with sets of outcomes that led to an impressive display of successful counting. We do not wish to diminish the remarkable work that these students accomplished. However, a closer look at the findings also reveals a number of unexpected phenomena that deserve discussion, including new insights into Lockwood's (2013) model.

An Overreliance on Patterning may Preclude the Development of the Multiplication Principle We previously emphasized that the multiplication principle is a fundamental aspect of counting, providing conceptual justification for why basic counting formulas work. Given these students' success, and given the fundamental role that the 
multiplication principle plays in counting, we expected the students' work to be characterized by rich and frequent justification via the multiplication principle. In terms of Lockwood's (2013) model, we anticipated that if students were to reinvent counting formulas successfully and meaningfully, they would use sets of outcomes first to reason about the counting process of the multiplication principle. Then, the students could reason about why particular products might be desirable and would reinvent notation to help them write those products more generally. We did not necessarily expect that students would be successful in this trajectory (nor that they would draw on outcomes), but we did feel that if students were able to reinvent the formulas, they would necessarily have developed at least some sense of the multiplication principle. This expectation was based both on our mathematical knowledge and our prior experience teaching and conducting research on students' counting (Fig. 17a shows the trajectory we expected going into the teaching experiment). However, our findings indicate that the students' work was, surprisingly, not based on the multiplication principle, but instead it was almost entirely based on empirical patterning (this actual trajectory is reflected in Fig. 17b). In the remainder of this subsection we provide evidence of this finding. To illustrate this point, we first revisit the students' work on the Quiz Questions problem and the Coin Flips problem.

Revisiting the Quiz Questions and Coin Flips problems As discussed, a natural approach to solving the Quiz Questions problem is to use the multiplication principle; we can argue that there are eight independent stages to the problem, each of which have four possibilities, and so the answer is $4^{8}$. We expected that Thomas and Robin might eventually reason similarly. Instead, however, the reader might recall that Thomas and Robin did not appear to think of constructing an outcome in stages by considering that there were four choices for each question, and they never seemed to reason using the multiplication principle. They arrived at the correct answer of $4^{8}$ by noticing how quickly things were growing (suggesting exponentiation) and verifying this guess by establishing a pattern (see Fig. 4). While they ultimately decided that the answer should be $4^{8}$, they went on to say that they did not see how else they could check their answer, noting "we can only go up to the third [column] without missing anything." There was not a sense that they could argue about what was happening in terms of why this answer might make sense more generally, aside from the pattern they had detected. We
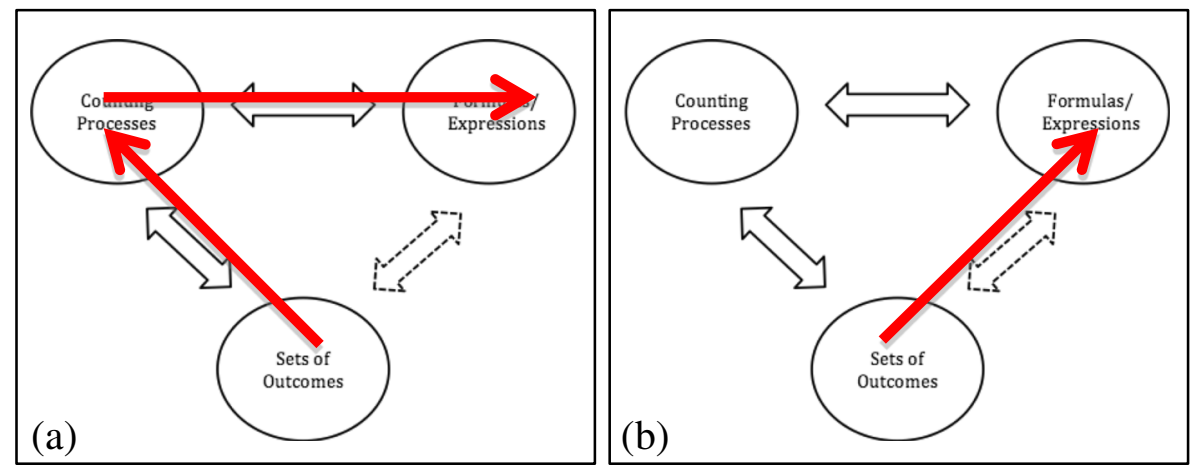

Fig. 17 Anticipated (a) and actual (b) trajectories of students' reasoning 
thus see in this problem that while they arrived at the correct answer of $4^{8}$, this answer was entirely based on a pattern they had empirically established through writing outcomes. They found four possibilities by listing outcomes, and then they found 16 by listing outcomes, and that information, combined with the focus on exponentiation, suggested to them that the answer was 4 to a power.

It is not the case that they reasoned about the number of options possible for each question, nor did they seem to have conceptual understanding of why the solution made sense. Notice that at no time did they make an argument (in the smaller cases or the original problem) that the answer was grounded in the multiplication principle (or reasoning consistent with the multiplication principle). This raises an important issue about justification in solving counting problems. Thomas had said about the Quiz Questions problem, "It's just going to be hard to check if we're right or not." While he is correct that actually empirically checking an answer can be difficult for counting problems, this language and their subsequent behavior on the problem suggests that they did not see any route to justification aside from pure empirical checking. We would argue that even an intuitive sense of the multiplication principle could go a long way toward providing some rationale behind their solution, but it was not something with which the students had facility.

We suggest that the students' use of outcomes and their ability to rely so heavily (and so successfully) on empirical patterns from their lists of outcomes may have precluded their developing the multiplication principle. It is not surprising that the students did not themselves reinvent a rigorous statement or definition of the multiplication principle, as this was not itself the focus of the teaching experiment. However, given the pervasiveness of the multiplication principle (as seen mathematically and also throughout our experiences with prior students), it surprised us that the students did not eventually develop and consistently use at least an informal argument that resembled the multiplication principle. We acknowledge that they did use the operation of multiplication at times during the teaching experiment, and on occasion (such as in the Bob \& Jim problem) their work may have been suggestive of the multiplication principle, but overall their counting activity was characterized by not having deep understanding of the multiplication principle to the point that they could use it flexibly as a tool.

Similarly, their work on the Coin Flips problem was consistent with the method they used throughout, in which they used sets of outcomes to construct an initial listing, which then motivated them to generalize based on numerical patterns. This approach was characteristic of their means of justification. They did not use the multiplication principle to justify why the product they were getting was the correct answer, but rather they argued based on the empirical patterns they had found. Our analysis suggests that the students did not show evidence of conceptual understanding of why these formulas might make sense, particularly related to the multiplication principle.

As the results demonstrate, this absence of the multiplication principle as a consistent tool is representative of Thomas and Robin's work on almost all of the problems that involved large numbers, throughout the entire teaching experiment. In fact, their reinvention of the formulas and their subsequent work on new tasks together suggest a similar, consistent method. The ability to match patterns and reason from sample outcomes was reliable for them, and their work on every subsequent problem provided repeated and consistent evidence for the fact that they did not use the multiplication 
principle in their reasoning. Accordingly, although they almost invariably eventually arrived at the correct answer by way of applying a correct formula (and using sets of outcomes to do so), their understanding of why the answer made sense felt, to us, incomplete.

New Insights into the Model The students' work also shed new light on Lockwood's (2013) model. In particular, prior to this study, students' struggles with counting problems might be frequently characterized by Fig. 18a, in which students commonly neglect sets of outcomes and instead look simply to match formulas or rely on memorized problem features. However, the students in our teaching experiment were not making these same kinds of mistakes. Instead, in terms of the model, we would characterize their work by the diagram in Fig. 18b. Our students did not display a grasp of the multiplication principle and they did not use a counting process like the multiplication principle to justify their work. Rather, they relied entirely on empirical patterning (similar to Harel's (2001) result pattern generalization).

Prior to this study, the relationship between sets of outcomes and formulas/ expressions was not well understood. Our findings thus make a theoretical contribution to the model, suggesting that patterning is an appropriate description for how to characterize the relationship between sets of outcomes and formulas/expressions. Moreover, the students in this study provide an existence proof that, given appropriate circumstances, the relationship between sets of outcomes and formulas/expressions can develop in students as a natural aspect of reasoning about counting.

Sets of Outcomes Are Valuable, but They Are Not Enough A number of studies (e.g., Author, Date, Date) have already shown that sets of outcomes are an important feature of combinatorial reasoning and can support good counting practices. Our findings support this claim. As we have indicated, these students productively reasoned about outcomes in almost every counting situation in which they found themselves - in formulating the answer to a problem in Phase 1, in developing patterns that contributed to generalization in Phase 2, and in determining which formula best fit their current situation in Phase 3. Looking at and arguing about outcomes was a fundamental aspect of their deciding which formulas might be appropriate, and this allowed them to avoid nonsensical answers and led them away from the temptation simply to apply the
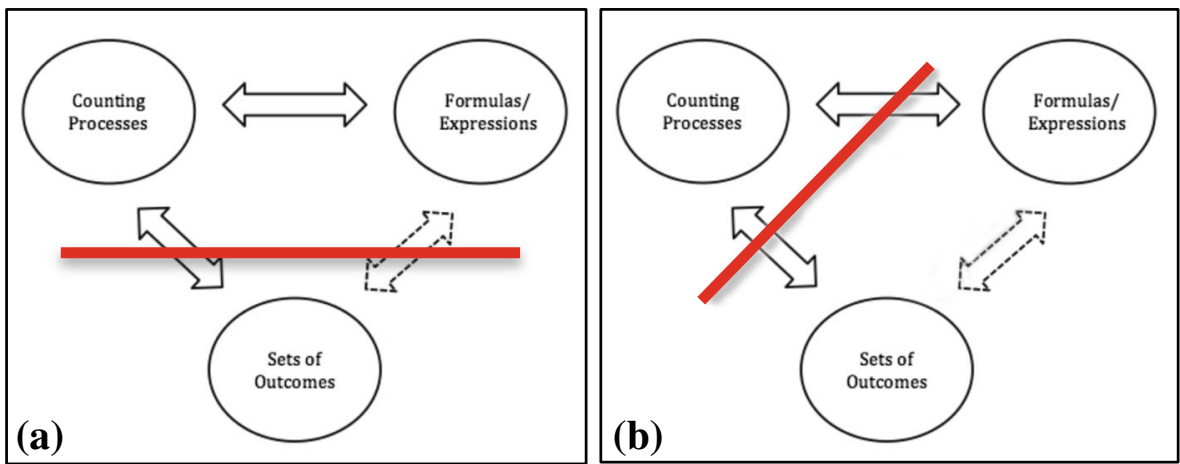

Fig. 18 A picture of the model depicting formula use (a) without sets and (b) with a heavy reliance on patterning 
formulas blindly. The study thus provides strong evidence for how useful focusing on outcomes can be in counting, particularly for novice counters.

However, this study also reveals that sets of outcomes are not sufficient in helping students make sense of and justify their counting activity, and we highlight two related potential issues with these students' attention to outcomes in absence of counting processes like the multiplication principle. First, these students showed that patterning may not lead to justification. If indeed we seek to have students who deeply understand their counting activity (which is a clear goal for developing proficient counters), we need to emphasize more than just patterning. Second, our study suggests that an overreliance on outcomes may actually inhibit the development and use of the multiplication principle, the understanding of which, as we have mentioned, is central to the entire subject. Therefore, while work with outcomes can be useful for students, caution should also be taken so students do not rely on outcomes to the point that they ignore deeper and more conceptual aspects of their work.

In summarizing the takeaways from the teaching experiment, then, there is a tension between two different aspects of the overall narrative that we can describe. On the one hand, these students were very successful. Given the rates at which students typically correctly solve counting problems, even in studies in which they have ample time to work on a problem, a $97 \%$ success rate is exemplary. Additionally, their ability to reinvent formulas, and their impressive facility with sets of outcomes are laudable. We want to celebrate these students' accomplishment in this regard and to present our ideas for why they were so successful. On the other hand, we do not feel that their high success rates tell the entire story, as there were also serious limitations to their approach. Specifically, because of their strong connection to the set of outcomes, and because this often enabled them to rely heavily on patterns, they did not encounter a need to develop strong multiplicative thinking to explain what they were doing. As a result, they often lacked the kind of robust reasoning and justification that would be expected of students who had deeply understood the concepts.

\section{Next Steps and Pedagogical Implications}

We acknowledge that our work presented in this paper is restricted to lengthy sessions with two above-average students, which is hardly a generalizable scenario. However, similar to other exploratory studies that have employed a teaching experiment methodology (e.g., Steffe and Thompson 2000; Swinyard 2011), we gain deep insight into student thinking by being able to spend a considerable amount of time closely examining students' combinatorial thinking and activity. Our work here provides a much needed foundation in our quest to understand aspects of students' combinatorial reasoning, and we argue that without having spent significant time with these students, we might not have gained such rich insights about their successes, struggles, activity, and reasoning, which ultimately helped to refine Lockwood's conceptual model (2013). Now that we are armed with these initial findings, there are a number of natural next steps in our program. First, we can try to determine whether our findings hold true more generally among other students. In particular, we may test whether we see the same kind of empirical patterning in other students, and we can try to evaluate more broadly the extent to which students draw upon and understand the multiplication principle. In 
addition to studying our findings more broadly, there are other avenues to pursue. Our study suggests that we need to continue to investigate more deeply the ways in which students connect their counting processes with their sets of outcomes, and we feel that the multiplication principle is a key aspect of this connection. Therefore, we want to investigate the principle itself, studying students' conceptualizations and development of it as a tool for counting. This may involve reinventing the multiplication principle specifically, which we believe may be more productive for students than having them simply reason about statements they are given or told. Indeed, we could then examine whether students who had reinvented the multiplication principle had more success with reinventing, understanding, and justifying their solutions to counting tasks using the four formulas discussed above.

In terms of pedagogical implications, again we acknowledge the narrow scope of our study and the fact that instructors cannot realistically spend ten hour-long sessions teaching counting to students. However, we suggest two implications for the consideration of those who teach counting problems. First, students are capable of reasoning about counting principles and formulas, and they may benefit from coming up with formulas on their own rather than being explicitly taught or told them. The time the students had to explore problems on their own before they reinvented the formulas appeared to be very beneficial to them, and they carefully considered outcomes and thought hard about the problem rather than blindly applying formulas. This suggests that some up front work (even just an activity or two) that allows students to engage with and perhaps even try to generalize their own formulas might be beneficial. Second, when students solve problems and try to decide which formula they should use, they should focus their attention on the nature of what is being counted. When the students in our study were tasked with using an existing formula, they were very successful in large part because they articulated an outcome and tied that outcome (and not key words) to the formula. Teachers should instruct students to use the nature of their outcome to determine what kind of problem/situation they are in and to use that information to decide which formula to use.

Ultimately, we want to move toward designed-based research that targets the development of instructional tasks and sequences that can help students be more conceptually grounded in their counting activity. Such research would follow the trajectories of researchers in other undergraduate content areas, such as differential equations (e.g., Rasmussen and King 2000) and abstract algebra (e.g., Larsen 2013). The teaching experiment reported in this paper has laid the groundwork for such subsequent investigations in which we can explicitly target the development of particular concepts and ideas (such as the multiplication principle, or sets of outcomes) that we feel might be important aspects of students' combinatorial thinking.

\section{References}

Annin, S. A., \& Lai, K. S. (2010). Common errors in counting problems. Mathematics Teacher, 103(6), 402409.

Batanero, C., Navarro-Pelayo, V., \& Godino, J. (1997). Effect of the implicit combinatorial model on combinatorial reasoning in secondary school pupils. Educational Studies in Mathematics, 32, 181-199. 
Cobb, P. (2000). Conducting teaching experiments in collaboration with teachers. In R. A. Lesh \& A. E. Kelly (Eds.), Handbook of research design in mathematics and science education (pp. 307-333). Hillsdale: Erlbaum.

Dubois, J. G. (1984). Une systematique des configurations combinatoires simples. Educational Studies in Mathematics, 15(1), 37-57.

Eizenberg, M. M., \& Zaslavsky, O. (2004). Students' verification strategies for combinatorial problems. Mathematical Thinking and Learning, 6(1), 15-36.

Fischbein, E., \& Gazit, A. (1988). The combinatorial solving capacity in children and adolescents. ZDM, 5, 193-198.

Freudenthal, H. (1973). Mathematics as an educational task. Dordrecht: Reidel.

Godino, J., Batanero, C., \& Roa, R. (2005). An onto-semiotic analysis of combinatorial problems and the solving processes by university students. Educational Studies in Mathematics, 60, 3-36.

Gravemeijer, K. (1998). Developmental research as a research method. In A. Sierpinska \& J. Kilpatrick (Eds.), Mathematics Education as a Research Domain: A Search for Identity (pp. 277-296). Dordrecht: Kluwer.

Hadar, N., \& Hadass, R. (1981). The road to solve combinatorial problems is strewn with pitfalls. Educational Studies in Mathematics, 12, 435-443.

Halani, A. (2012). Students' ways of thinking about enumerative combinatorics solution sets: the odometer category. In S. Brown, S. Larsen, K. Marrongelle, \& M. Oehrtman (Eds.), Proceedings for the Fifteenth Special Interest Group of the MAA on Research on Undergraduate Mathematics Education (pp. 59-68). Portland: Portland State University.

Harel, G. (2001). The development of mathematical induction as a proof scheme: a model for DNR-based instruction. In S. Campbell \& R. Zaskis (Eds.), Learning and Teaching Number Theory, Journal of Mathematical Behavior (pp. 185-212). New Jersey: Ablex Publishing Corporation.

Hiebert, J., \& Lefevre, P. (1986). Conceptual and procedural knowledge in mathematics: an introductory analysis. In J. Hiebert (Ed.), Conceptual and procedural knowledge: The case of mathematics (pp. 1-27). Hillsdale: Lawrence Erlbaum.

Larsen, S. (2013). A local instructional theory for the guided reinvention of the group and isomorphism concepts. Journal of Mathematical Behavior, 32(4), 712-725. doi:10.1016/j.jmathb.2013.04.006.

Lockwood, E. (2011a). Student Approaches to Combinatorial Enumeration: The Role of Sets of Outcomes. Unpublished Doctoral Dissertation. Portland State University.

Lockwood, E. (2011b). Student connections among counting problems: an exploration using actor-oriented transfer. Educational Studies in Mathematics, 78(3), 307-322. doi:10.1007/s10649-011-9320-7.

Lockwood, E. (2013). A model of student's combinatorial thinking. Journal of Mathematical Behavior, 32, 251-265. doi:10.1016/j.jmathb.2013.02.008.

Lockwood, E. (2014a). Both answers make sense! Using the set of outcomes to reconcile differing answers in counting problems. Mathematics Teacher, 108(4), 296-301.

Lockwood, E. (2014b). A set-oriented perspective on solving counting problems. For the Learning of Mathematics, 34(2), 31-37.

Lockwood, E., \& Gibson, B. (2014). The value of systematic listing in correctly solving counting problems. In T. Fukuwa-Connelly, G. Karakok, K. Keene, and M. Zandieh (Eds.), Proceedings for the Seventeenth Special Interest Group of the MAA on Research on Undergraduate Mathematics Education. Denver: Northern Colorado University (pp. 153-168).

Lockwood, E., Swinyard, C. A., \& Caughman, J. S. (In press). Modeling outcomes in combinatorial problem solving: the case of combinations. To appear in the Proceedings for the Eighteenth Special Interest Group of the MAA on Research on Undergraduate Mathematics Education. Pittsburg: West Virginia University.

Maher, C. A., Powell, A. B., \& Uptegrove, E. B. (Eds.). (2011). Combinatorics and Reasoning: Representing, Justifying, and Building Isomorphisms. New York: Springer.

Martin, G. E. (2001). The Art of Enumerative Combinatorics. New York: Springer.

Mazur, D. R. (2009). Combinatorics: A Guided Tour. Washington, DC: MAA.

Oehrtman, M., Swinyard, C., \& Martin, J. (2014). Problems and solutions in students' reinvention of a definition for sequence convergence. Journal of Mathematical Behavior, 33, 131-148.

Piaget, J., \& Inhelder, B. (1975). The origin of the idea of chance in children. New York: W. W. Norton \& Company, Inc.

Rasmussen, C., \& King, K. (2000). Locating starting points in differential equations: a realistic mathematics approach. International Journal of Mathematical Education in Science and Technology, 31, 161-172.

Richmond, B., \& Richmond, T. (2009). A Discrete Transition to Advanced Mathematics. Providence: American Mathematical Society.

Steffe, L. P., \& Thompson, P. W. (2000). Teaching experiment methodology: Underlying principles and essential elements. In R. Lesh \& A. E. Kelly (Eds.), Research design in mathematics and science education. Mahwah: Lawrence Erlbaum Associates. 
Swinyard, C. (2011). Reinventing the formal definition of limit: the case of Amy and Mike. Journal of Mathematical Behavior, 30, 93-114.

Tall, D., \& Vinner, S. (1981). Concept image and concept definition in mathematics with particular reference to limits and continuity. Educational Studies in Mathematics, 12(2), 151-169.

Tucker, A. (2002). Applied Combinatorics (4th ed.). New York: John Wiley \& Sons. 